\title{
The influence of basketball shooting Angle afor shooting
}

\author{
Weiqi Jiang \\ Institute of Physical Education, Huanggang Normal University, Huangzhou 438000, China
}

\begin{abstract}
Shooting technique is correct standards directly affect the shooting, to improve the shooting, must fully grasp of the basic elements of shooting skills. Reference documents, this article USES theoretical analysis, comparative analysis and other methods, according to the theory of basketball shooting skills summarized the influence of basic elements for shooting, and mainly through mechanics analysis, theoretical analysis, comparative analysis, and other key means to shoot the ball shoot height, Angle for shooting the ball, aiming point, and the influence of ball rotation to analyze the influence of the shooting, from theory to explore the basic factors affecting shooting, for basketball education and provide theoretical basis for the practical application of athletes.
\end{abstract}

\section{Introduction}

Basketball game is a scoring game decided in a competing against skill of sports. Score, is the ultimate pursuit of score in the game, so the discretion of the shooting to a certain extent determines the outcome of competition and irreplaceable position in the basketball game, each athletes and coaches have been also one of the most concerned problems.

Study of how to improve the shooting has not interrupted, the domestic some scholars put forward its own views on how to improve the shots and theory made certain contribution for our country's basketball career, including: $\mathrm{Xu}$ Yan, such as using literature method, observation method and biomechanics analysis of basketball player shooting technology for $3 \mathrm{~d}$ video and parsing, analyzes the main movement joint movement characteristics of athletes, shooting hand movement, and movement characteristics of the ball. Research shows that only when shooting athletes deal with onset of coordination force, the point height and Angle, and the relationship between the balls to instant action can improve shot shot [1-4]. WenZhongBo in the introduction to the basketball game of shooting athletes psychological quality are pointed out in the influence of psychological impact on shooting in the game, and puts forward several athletes psychological training methods, and promote open for athletes and college students' psychological counseling theory [2-5].

Basketball shooting skills will be the basic elements of the impact on the shooting, and mainly adopts mechanics analysis, theoretical analysis, comparative analysis to the shot release height and Angle to the shooting, aiming point, and the influence of ball rotation analysis of shooting effect. In theory and put forward some advice in competition and training are practical.

\section{Basketball hit rate important influential factors mechanical analysis}

In reality, shooting can be divided as one-hand shot and two-hand shot two types. One-hand shot is relative general, while two-hand shot mostly appears in women's middle and far distance shooting. Firstly, practical application tells us that two-hand shot consumes longer time than one-hand shot, it nearly is $0.1-0.3$ seconds more, adopt two-hand shot is very bad in basketball such intense competition technological game. Secondly, two-hand shot mostly puts the ball right above the forehead, it is prone to obscure shooters' visions, affects shooters perception and judgment on surrounding environment, and it is difficult to change and prone to be block shot by opponents. According to above two reasons, the writer suggests to adopt one-hand shot.

\subsection{Rebound aiming point mechanical basis}

Momentum conservation law is rebound aiming point shot mechanical principle, if ignore basketball and rebound energy loss, then basketball before and after hitting rebound included angle with rebound are approximately the same, adding that hoop area $\left(6358.516 \mathrm{~cm}^{2}\right)$ is around fifteen times basketball area $\left(415.265 \mathrm{~cm}^{2}\right)$, so aiming points allows certain error, the error needs to be grasped by athlete with accurate sports perception through numerous training.

By investigation, aiming point in the heart of athlete has five types: 1) hoop front edge; 2) hoop back edge; 3) imaginary hoop central point; 4) imaginary hoop center line point right above $20 \mathrm{~cm}$; 5) the whole hoop. Here mentioned front edge, back edge take athlete index finger as reference point.

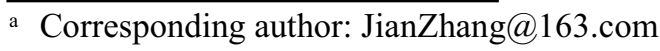




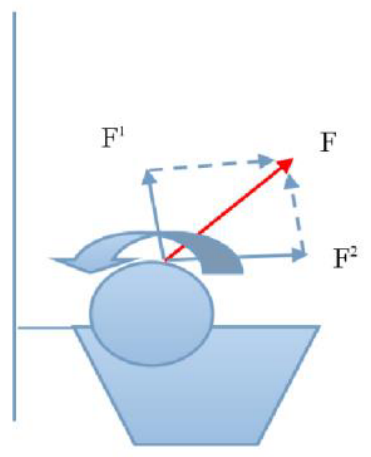

Figure 1. Force situation of Forward spin contacting with hoop.

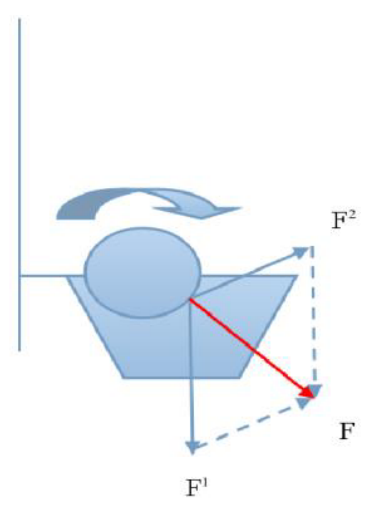

Figure 2. Force situation of back spin contacting with hoop.

When shooting, it is back spin; the writer thinks aiming point should be hoop back edge. If it takes hoop front edge as aiming point, it will occur to three cases: 1) Shooting exertion is slightly small, ball doesn't contact with hoop; 2) Ball just touches hoop, due to it is back spin, it will be popped up from hoop after touching hoop; 3) Shooting force is slightly bigger, ball touches back edge of hoop and pops into hoop. According to above analysis, when aiming point is hoop front edge, hit rate is $33.3 \%$. There are three cases when aiming point is hoop back edge: 1) Shooting exertion is slightly small, ball doesn't contact with hoop back edge and drops into hoop; 2) basketball just touches hoop back edge, ball rebounds into hoop 3) When shooting, ball excessive deflects from hoop, ball cannot enter into hoop. According to above analysis, hit rate on the condition that aiming point is hoop back edge is $66.7 \%$. When aiming point locates in other three cases, due to imaginary points are not easy to aim when shooting, it is not fit for shooting aiming point.

When take hoop back edge as aiming point, ball forward spin and back spin also have certain impacts on hit rate. By analyzing, field goal percentage by back spin suffered resultant force after ball contacting with hoop is larger than forward spin. Therefore author suggests that it should try to let ball to fast back spin when shoot in middle and far distances, so that will improve hit rate as Figure 1 and Figure 2.

\subsection{Ball rotational mechanical analysis}

When shooting, after basketball releasing, basketball suffers gravity, air buoyancy and air resistance three kinds of forces when moving in the air, air buoyancy and air resistance are very little with respect to gravity that can be ignored, so it can think that basketball air motion trajectory is oblique projectile motion, oblique projectile motion each parameter is up to sum total of each kind of element that shooters shooting instant before releasing ball and releasing instantaneous act on ball, its trajectory:

$$
H=v \times \sin \theta / 2 g \quad L=v \times \cos \theta \times v \times \sin \theta / g
$$

Shooting motion let basketball to rotate in the air, so basketball motions in the air is c compound movement that oblique projectile and basketball surround sphere frontal axis to make rotation, therefore shooting motions basketball movement trajectory is different from general oblique projectile movement. Basketball back spin is generated when shooting, shooters lower limbs pedal the ground, waist, abdomen, and upper limbs by virtue of each joint chain transmitting, ball releasing instant wrist bends forward, index finger acting on the back bottom part of basketball [6].

Basketball back spins effects and mechanical analysis in shooting:

1)High speed back spin can effective overcome air resistance, it is beneficial to keep basketball relative stability in the air, which is very important for middle and far distances shooting motions. When basketball moves slowly in the air, air to sphere surface friction force is smaller, basketball spherical surface and adjacent airflow have a small gap, and we call it spherical surface layer. The existence of spherical surface layer lets sphere moving direction front and back regions to have insignificant pressure differences, airflow still can keeps steady airflow after passing through basketball it has little impacts on basketball. When shooting in middle and far distances, basketball speed is larger, basketball surface and airflow friction force increase, and causes spherical surface layer being damaged, when airflow gets through sphere peeling point (as Figure 3, F1 is stress, F2 is lift force, $\mathrm{M}$ point is peeling point, $\mathrm{V}$ is ball speed), it generates a flow line that has larger differences with original airflow, let sphere back region to generate larger laminar flow eddy zone, basketball front and back pressure differences are larger that cause sphere suffering resistance so that it diminishes basketball running height and distance, which is bad for middle and far distances. If basketball back spins at high speed when shooting in middle and far distances, due to air exists viscosity, basketball surface is not smooth, when basketball spins, it drives basketball surface adjacent gas particle to generate a puff of air circulation that in the same direction of basketball spinning direction. Air circulation and airflow make interactive effects and can weaken sphere front and back laminar flow eddy, it causes basketball surrounding airflow changing, effective diminishing air to ball resistance, it is beneficial to control basketball running trajectory and improve hit rate.

2) High speed back spinning basketball can let basketball to suffer a lift force( refer to Figure 3 F2), basketball lift force existence can enlarge basketball movement radian, increase basketball incident angle, enlarge hoop exposed surface and improve field-goal 
percentage. Therefore, it is clear due to basketball back spinning existence, it causes basketball upper side airflow speed to be big but flux is small, while upper side airflow speed is small but flux is big by unequal basketball up and down airflow volume and flow speed. According to Bernoulli's equation : $P+\rho g h+1 / 2 \rho v 2=$ constant,$\rho$ is air density, $g$ is gravity accelerated speed, the two are constant values, $h$ is fluid height, $h$ changes can be ignored with respect to atmospheric height. Therefore, it is clear that $v$ and $P$ are in inverse proportion, theoretically shooting distance gets further, basketball release speed would be faster. Flying basketball upper side (as Figure 4's A point) with respect to lower side(as Figure 4's B point) point is low pressure region, therefore it generates a upward lift force for basketball, pressure differences get bigger then basketball lift force would get bigger, flying basketball parabola radian would get bigger, radian gets bigger and then incident angle would get bigger, incident angle gets bigger then basketball exposed area will get bigger so that increase basketball odds of entering into hoop. By above analysis, it is clear that shooting instant high speed back spinning can improve hit rate.

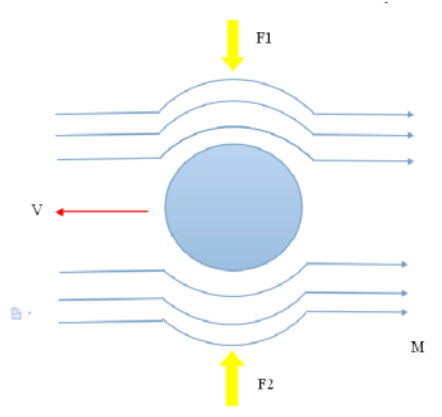

Figure 3. Magnus effect.

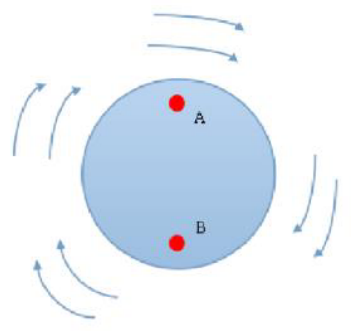

Figure 4. Back spin up and down two terminal speed pressures.

Basketball forward spinning generation generates due to shooting motion when ball released, shooters index finger acts on the front bottom part of basketball, forward spinning effects and mechanical analysis are as following: Forward spin similarly is beneficial to basketball to keep its relative stability when flying in the air, causes can refer to 2.5.2 relative statements; When make quick attacking, it suggests to use forward spin to act on similar force to ball, for basketball speed, forward spin is the fastest, non-spinning ball is secondly, back spin is the slowest, while basketball flight distance and radian size as well as speed size ranking orders are on the contrary. Though forward spin will suffer downward pressure in flight, considering forward spin are mostly used in close range shooting, so its influence can be ignored.

\section{Shooting release height and release angle mechanical analysis}

\subsection{Basic conditions for basketball shooting into hoop}

When shooting, incident angle size decides ball hit rate, according to shooting instant, basketball parabola, we know that incident angle is up to release angle when shooting. To easy to understand, we adopt geometric graphics to analyze, as Figure 5 shows, $\mathrm{AB}$ is hoop diameter $(0.45 \mathrm{~m}), \mathrm{CD}$ is basketball diameter $(0.25 \mathrm{~m})$, here we assume that basketball flies in the horizontal direction, the hoop rotates upward $\mathrm{X}$ angle to $\mathrm{EF}$ with a center of a circle, by Figure 5, it is clear that EG must be larger than $\mathrm{CD}$ so as to let basketball to enter into hoop:

$$
\operatorname{Sin} \alpha=\frac{E G}{E F}=\frac{0.25}{0.45}
$$

According to calculation, it is clear that basketball minimum incident angle $\alpha=33^{\circ} 45^{\prime}$, incident angle must be larger than $33^{\circ} 45^{\prime}$, and then basketball can be shot into the hoop.
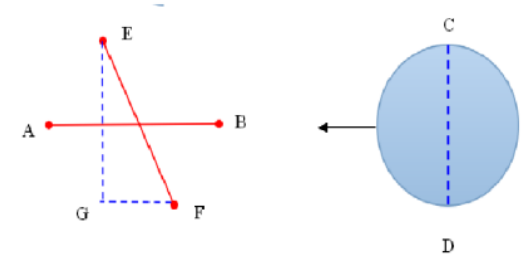

Figure 5. Incident angle when ball enters into the hoop.

\subsection{Influence analysis of hit rate when incident angle is unchanged, projection angle diminishes}

According to analysis, when height as $1.83 \mathrm{~m}$ shooter makes set shot, release height is in $2.1 \mathrm{~m}-2.2 \mathrm{~m}$, when makes jump shot, release height is in $2.7 \mathrm{~m}-2.9 \mathrm{~m}$, when shooter is in shooting distance $5 \mathrm{~m}$, incident angle $33^{\circ} 45^{\prime}$, according to above formula(5) calculation, it can get set shot projection angle $\varphi=45^{\circ} 18^{\prime}$, when makes jump shot, projection angle ${ }_{\phi}{ }^{\prime}=37^{\circ} 33^{\prime}$.Jump shot release height is higher than that of set shot, and basketball flying parabolic trajectory is relative shorter in the air, basketball suffered external force impacts are smaller, so jump shot is relative stable by comparing to set shot.

\section{Conclusion}

In this paper, the technological factors affecting the rate of shooting in basketball are analyzed and expounded, and the important technical factors affecting shooting mechanics analysis, through the analysis we know that 
after the ball after the ball in contact with the basket of resultant force make the basketball hit probability compared with the former more likely, therefore when long distance shooting in should try to make the ball after high speed spinning, it will improve the hit rate. In addition we also focus on emergence Angle and incident Angle of mechanics analyses the influence of shooting, hoping to provide reference to coaches and athletes [7].

\section{References}

1. J. H. Li, Journal of Shenyang Sport University, 25, 63-66(2006).
2. L. N. Wang et al, China Sport Science, 19, 37-40(1999).

3. J. Mao, S. G. Shan, Journal of Wuhan Institute of Physical Education, 46, 70-73, 87(2012).

4. L. Zhang, China Sport Science and Technology, 42, 50-52(2006).

5. F. F. Fu, Z. F. Yu, Q. N. Zhang. Shandong Sports Science \& Technology, 28, 24-25(2006).

6. G. V. da Silva, M. Muler, M. F. V. Prado et al. Journal of Coastal Research, 2016.

7. H. Huang, Z. L. Liu, International Conference on Engineering And Business Management, 1-8, 5186-5189(2010). 